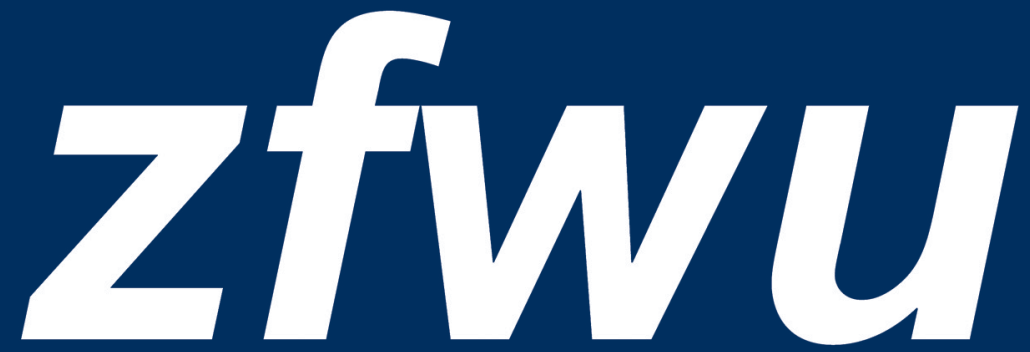

Zeitschrift für Wirtschafts- und Unternehmensethik

Journal for Business, Economics \& Ethics

\title{
Unternehmensverantwortung in historischer Perspektive
}

Rainer Hampp Verlag ISSN 1439-880X (print) 


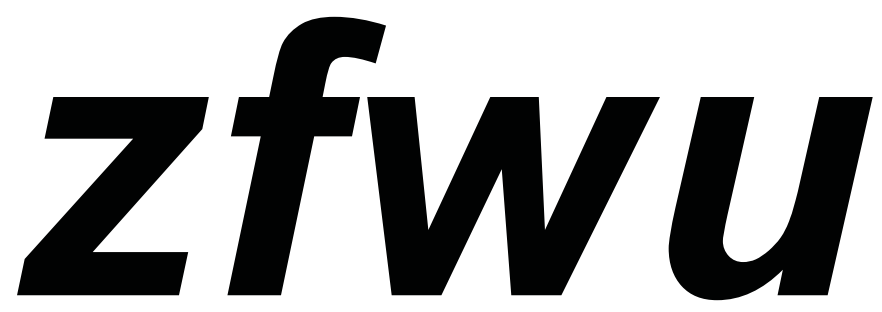

Zeitschrift für Wirtschafts- und Unternehmensethik

Journal for Business, Economics \& Ethics

herausgegeben von

Prof. Dr. Thomas Beschorner (St.Gallen, Schweiz)

Prof. Dr. Dr. Alexander Brink (Bayreuth und Witten/Herdecke)

PD Dr. Bettina Hollstein (Erfurt)

Dr. Marc C. Hübscher (Hamburg)

Dr. Olaf J. Schumann (Frankfurt a.M.)

\section{Wissenschaftlicher Beirat}

Prof. Dr. Michael S. Aßländer (Zittau)

Dr. Dr. Thomas Bausch (Berlin)

Prof. Dr. Georges Enderle (Notre Dame, USA)

Prof. Dr. Eilert Herms (Tübingen)

Prof. Dr. i.R. Hans G. Nutzinger (Kassel)

Prof. Dr. Fabienne Peter (Warwick, UK)

Prof. Dr. Reinhard Pfriem (Oldenburg)

Prof. Dr. Birger P. Priddat (Witten/Herdecke)

Prof. Dr. Andreas Suchanek (Leipzig)

Prof. em. Dr. Peter Ulrich (St.Gallen, Schweiz)

Prof. Dr. Dr. Johannes Wallacher (München)

Prof. Dr. Josef Wieland (Friedrichshafen)

\section{Berliner Forum}

Rainer Hampp Verlag ISSN 1439-880X 


\section{Impressum}

Zeitschrift für Wirtschafts- und

Unternehmensethik (zfwu)

ISSN 1439-880X

\section{Anschrift}

c/o Universität St. Gallen

Institut für Wirtschaftsethik

Girtannerstrasse 8

CH-9010 St. Gallen

c/o Universität Bayreuth

Institut für Philosophie

Universitätsstraße 30

D-95447 Bayreuth

Tel.: +49-(0)921-554148

E-Mail: redaktion@zfwu.de

www.zfwu.de

\section{Herausgeber der zfwu}

Prof. Dr. Thomas Beschorner

(St. Gallen, Schweiz)

Prof. Dr. Dr. Alexander Brink

(Bayreuth und Witten/Herdecke)

PD Dr. Bettina Hollstein (Erfurt)

Dr. Marc C. Hübscher (Hamburg)

Dr. Olaf J. Schumann (Frankfurt a.M.)

\section{Herausgeber dieser Ausgabe}

Thomas Hajduk (St. Gallen, Schweiz) Prof. Dr. Thomas Beschorner (St. Gallen, Schweiz)

\section{Redaktion}

Felix Meyer (Bayreuth)

Dana Sindermann (St. Gallen, Schweiz)

Thordis Taag (Heidelberg)

\section{Verlag}

Rainer Hampp Verlag

Marktplatz 5

D-86415 Mering

Tel.: +49-(0)8233-4783

Fax: +49-(0)8233-30755

Rainer_Hampp_Verlag@zfwu.de

\section{Bezugsmöglichkeiten}

Das Jahresabonnement (3 Ausgaben:

Februar/März, Juni/Juli und Oktober/November) kostet EUR 60,00 inkl. Mehrwertsteuer und Versand. Studenten erhalten gegen jährliche Vorlage der Studien-bescheinigung 50\% Ermäßigung. Der Bezug aus dem Ausland ist um EUR 10,35 teurer. Das Einzelheft kostet EUR 24,80 frei Haus.

\section{Copyright}

Alle Rechte vorbehalten.

\section{Hinweise für AutorInnen}

Manuskripte sind, sofern es sich um Erstveröffentlichungen handelt, der Redaktion jederzeit willkommen. Dazu möchten wir Sie bitten, Ihren Text vorher in die $\mathbf{z f w u}$-Formatvorlage zu bringen (erhältlich bei der Redaktion und auf unserer Homepage) und ihn anschließend per E-Mail zu senden an: redaktion@zfwu.de Jeder Beitrag unterliegt einem doppelt verdeckten Gutachterverfahren. Publikationssprachen sind Deutsch und Englisch.

Weitere Informationen und Autorenhinweise unter:

www.zfwu.de 


\section{Editorial}

Unternehmensverantwortung hat eine lange Vergangenheit, aber noch wenig Geschichte. Denn während die Frage nach dem ethischen Gehalt des unternehmerischen Handelns Jahrhunderte, womöglich Jahrtausende zurückreicht, steckt die Historisierung von Unternehmensverantwortung noch weitgehend in den Kinderschuhen und findet wenig Beachtung in unternehmenstheoretischen und -ethischen Diskussionen. Dabei kann gerade der Blick in die Vergangenheit die konzeptionelle Vielfalt des Phänomens erhellen, seine zeitliche Wandelbarkeit herausstellen und durch das Aufzeigen von Kontinuitäten wie Brüchen aktuelle Diskussionen besser verstehen lassen.

Um der Geschichte von Unternehmensverantwortung mehr Sichtbarkeit zu verleihen und damit eine aus unserer Sicht längst überfällige Diskussion anzustoßen, widmet sich die vorliegende Ausgabe der $\mathbf{z} f \boldsymbol{w} \boldsymbol{u}$ dem Thema „Unternehmensverantwortung in historischer Perspektive“. Wir freuen uns sehr, dass mit Frederick Bird, Kenneth E. Goodpaster, Gabriel Abend und Kenneth J. Lipartito vier prominente Vertreter aus dem anglo-amerikanischen Sprachraum für eine solche Perspektive als Autoren für die beiden Hauptbeiträge und die dazugehörigen Korreferate für diese Ausgabe gewonnen werden konnten.

Das Thema Unternehmensverantwortung, so zeigen die Einreichungen und die Beiträge zu dieser Ausgabe, wird sowohl von Wirtschaftsethikern als auch von Historikern als relevantes Forschungsfeld wahrgenommen. Zugleich war und ist jedoch festzustellen - und manch ein Leser wird dies bereits an den verschiedenen Zitationsstilen erkennen -, wie unterschiedlich die Zugänge und Standards in den beiden genannten Disziplinen sind. Der interdisziplinären Ausrichtung der Ausgabe folgend wurden Beiträge im Idealfall von je einem Vertreter der Wirtschaftsethik und den Geschichtswissenschaften begutachtet. Hierbei war zu beobachten, dass die Gutachter teils stark konträr bewerteten. An der Beurteilung des Forschungsstands, der theoretischen Einbettung und dem Umgang mit Quellen wurden disziplinäre Grenzen sehr deutlich sichtbar.

Wir sehen diese Ausgabe daher als ein Forum für (Pionier-)Arbeiten zur Historisierung von Unternehmensverantwortung wie auch als Impuls für die weitere Forschung. Dafür sind interdisziplinäre Beiträge aus der Wirtschaftsethik wie aus den Geschichtswissenschaften und weiterer Fächer wünschenswert, die in den kommenden Jahren hoffentlich in Zusammenarbeit entstehen. Die Beiträge in diesem Heft zeigen aus unserer Sicht das große Potenzial, das diesem noch jungen Forschungsfeld innewohnt.

Wir eröffnen die vorliegende Ausgabe mit der paradigmatischen Frage, die Frederick Bird (Waterloo, Kanada) in seinem Hauptbeitrag stellt: Warum sollten diejenigen, die sich gegenwärtig mit Unternehmensethik beschäftigen, zurück in die Geschichte blicken? In seiner Antwort identifiziert Bird drei aus seiner Sicht typische Ausdrucksformen unternehmensethischer Praxis in der Geschichte, nämlich die Kultivierung von Tugenden, die Evolution der ethischen Problemlösungsfähigkeiten von Unternehmen und die Genese öffentlicher Standards. Von ihnen, so der Autor, könnten Unternehmen auch zur Lösung gegenwärtiger Krisen lernen. Kenneth Goodpaster (St. Thomas, Minneapolis) kommentiert Birds Überlegungen in seinem Korreferat. 
Ausgangspunkt des zweiten Hauptbeitrags von Gabriel Abend (New York) bildet die Geschichte der U.S.-amerikanischen Unternehmensethik, die zwar vordergründig von normativen Kontinuitäten und Stabilitäten geprägt sei, aber auf einer dahinter liegenden Ebene Variationen und Diskontinuitäten unterliege. Es sei dieser „moralische Hintergrund“, der unser Verständnis von Gesellschaft, Moral und den moralischen Grundlagen kapitalistischer Gesellschaften erkläre. An der Abgrenzung von Moral führt Abend seine Überlegungen aus. Kenneth Lipartito (Miami) übernimmt das Korreferat dieses Hauptbeitrags.

Michael S. A Bländer (Dresden/Zittau) untersucht in seinem Beitrag die Geschichte der deutschsprachigen Wirtschaftsethik, die bisher häufig als verspätete Antwort auf die US-amerikanische Wirtschaftsethikdebatte gesehen worden sei. Der Autor eröffnet eine alternative Perspektive, in der Entwicklungen und Themenfelder einer deutschsprachigen Wirtschaftsethikdiskussion ins Licht gerückt werden, welche wiederum bis zum Beginn des 20. Jahrhunderts zurückreichen. Florian Schui (St. Gallen, Schweiz) nimmt hierzu Stellung.

Martin Kolmar und Thomas Beschorner (beide St. Gallen, Schweiz) stellen in ihrem Beitrag die Frage nach dem Verhältnis von individueller, organisationaler und institutioneller Verantwortung, welches aus ihrer Sicht in vielen Disziplinen einseitig bestimmt werde. Vor diesem Hintergrund entwickeln sie in Anknüpfung an die Transaktionskostentheorie einen Ansatz, der die Zuweisung von Verantwortung als substanzielles Element einer ethischen Theorie versteht. Ingo Pies (Halle) trägt das Korreferat bei.

Das Moment der Verantwortung kehrt auch in dem zweiten Beitrag von Frederick Bird (Waterloo, Kanada) wieder, in dem der Historiker zu zeigen versucht, wie Unternehmensethik in verschiedenster Weise von einem sorgfältigen Blick in die Geschichte profitieren kann. Dieser 2009 veröffentlichte Beitrag hat früh den Wert historischer Perspektiven in der Wirtschaftsethik herausgearbeitet und wird daher in der vorliegenden Ausgabe leicht überarbeitet wiederveröffentlicht.

Eine zeitgeschichtlich-politische Perspektive verfolgen Felix C. Schweren (London) und Alexander Brink (Bayreuth und Witten/Herdecke) in ihrem Praxisbeitrag. Darin zeichnen sie die Entstehung der umstrittenen „CSR-Richtlinie“ der Europäischen Union nach. In seinem Korreferat ergänzt Thomas Loew (Berlin) den Beitrag durch eigene Überlegungen.

Wir schließen die Ausgabe mit drei Rezensionen von Peter Seele (Lugano, Schweiz), Alex Gertschen (Marburg) und Thomas Hajduk (St. Gallen, Schweiə).

Wir wünschen Ihnen eine wie hoffentlich immer anregende Lektüre der Beiträge dieser Ausgabe.

Thomas Hajduk und Thomas Beschorner 


\section{UNTERNEHMENSVERANTWORTUNG IN \\ HISTORISCHER PERSPEKTIVE}

hrsg. von Thomas Hajduk und Thomas Beschorner

Hauptbeitrag

Learning from History - The Relevance of the History of Business

Ethics for the Practice of Business Ethics

Frederick Bird

Warum sollten diejenigen, die sich gegenwärtig mit Unternehmensethik befassen, ein breiteres Verständnis davon anstreben, wie man in der Vergangenheit Unternehmensethik praktizierte? Der Essay verfolgt diese Frage, indem er in weiten historischen Strängen drei typische Ausdrucksformen unternehmensethischer Praxis untersucht: Bemühungen (1) geeignete Tugenden zu kultivieren, (2) die ethischen Problemlösungsfähigkeiten von Unternehmen zu erweitern und (3) öffentlich durchsetzbare normative Standards und Gesetze zu schaffen. Mit Blick auf sieben tiefe, gegenwärtige ethische Krisen schließt der Essay, indem er Wege auslotet, wie Unternehmen von einer kritischen Prüfung der Geschichte von Unternehmensethik lernen, um die damit verbundenen Probleme verantwortungsvoller anzugehen.

Korreferat

Varieties of Relevance

Kenneth E. Goodpaster

Hauptbeitrag

\section{How to Tell the History of Business Ethics}

Gabriel Abend

Die Geschichte der U.S.-amerikanischen Unternehmensethik und CSR weist einige wichtige normative Kontinuitäten und Stabilitäten auf. Ich argumentiere, dass diese normative Kontinuitäten und Stabilitäten mit Diskontinuitäten und Variationen eines moralischen Hintergrunds unterlegt sind. Erstere nenne ich Stabilitäten „,erster Ordnung", letztere Variationen ,zweiter Ordnung“. Die Ebene zweiter Ordnung oder des moralischen Hintergrunds ist der Ort des Geschehens - zumindest desjenigen Geschehens, das unser Verständnis von Gesellschaft, Moral und den moralischen Grundlagen kapitalistischer Gesellschaften am besten ausleuchtet. Anschließend gehe ich auf eine Facette des moralischen Hintergrunds ein, die für das wissenschaftliche Verständnis von Unternehmensethik und CSR besonders interessant sein könnte: Die Abgrenzung von Moral. Wie werden Grenzen zwischen moralischen und nicht-moralischen Dingen gezogen? Wie werden sie institutionalisiert und kontrolliert? Wie variieren sie über Zeit und Raum? Und ich zeige auch, warum diese empirischen Fragen konzeptuell kniffeliger sind als es auf den ersten Blick erscheinen mag. 
Korreferat

Business Ethics - A Historian's Perspective

Kenneth J. Lipartito

Beitrag

„Kapitalismus ist Gewinnsucht, die sich nicht schämt“Frühe Schriften zur Wirtschaftsethik im deutschsprachigen Raum

\section{Michael S. Aßländer}

Gemeinhin wird die deutschsprachige Debatte um eine Wirtschafts- und Unternehmensethik als eine verspätete Antwort auf die US-amerikanische Wirtschaftsethikdiskussion gesehen. Insbesondere sind es Autoren wie Karl Homann, Peter Ulrich oder Horst Steinmann und Albert Löhr, die das Thema Wirtschaftsethik ab den späten 1980er Jahren in Deutschland populär zu machen suchten. Wie im Folgenden zu zeigen sein wird, reichen die Wurzeln der deutschsprachigen Wirtschaftsethik jedoch bis zu Beginn des 20. Jahrhunderts zurück. Ziel des vorliegenden Beitrages ist es, die Entwicklung und die Themenfelder dieser frühen WirtschaftsethikDebatte in Deutschland darzustellen.

Korreferat

114 Deutschsprachige Wirtschaftsethik im Rückblick

Florian Schui

Beitrag

Locating Responsibility - An Extended Transaction Cost Approach Martin Kolmar und Thomas Beschorner

Die meisten Debatten in der Ethik, der Wirtschaftsethik und der normativen Ökonomik gehen von der oft impliziten Annahme aus, dass die Ebene normativer Verantwortlichkeit vorgegeben ist, so dass sie entweder den Individuen (Theorien der Moral), oder einer bestimmen institutionellen Ebene wie dem Staat (Gerechtigkeitstheorien) oder dem Unternehmen (Theorien der Unternehmensverantwortung) zufällt. Wir argumentieren, dass dieser Ansatz zu beschränkt ist, um einen umfassenden Blick auf die Lage zu bekommen, wie sich das Verhältnis individueller, organisationaler und institutioneller Verantwortung ergibt. Dazu entwickeln wir einen Ansatz, der die Zuordnung von Verantwortung als einen integralen Bestandteil einer ethischen Theorie erlaubt. Dieser Ansatz basiert auf Transaktionskosten als basale Erklärungsvariable. 
Korreferat

Taking or Locating Responsibility? - An Ordonomic Perspective

Ingo Pies

Beitrag

Why the Practice of Business Ethics Calls for a Due Regard for History

Frederick Bird

In diesem Essay werde ich vier Gründe untersuchen, warum ein sorgfältiger Blick in die Geschichte für Unternehmensethik wichtig ist: (1) um lokale kulturelle Normen und Praktiken schätzen zu lernen; (2) um zu verstehen, wie gegenwärtige Probleme entstanden sind und welche Verantwortung für die Vergangenheit besteht; (3) um einen lebhaften Sinn dafür zu entwickeln, was jetzt möglich ist und was nicht, und wann welche Art von Handlungen gefragt sind; und (4) um zu beobachten, wie bestehende Praktiken sich über die Zeit entwickelt haben.

Praxisbeitrag

CSR-Berichterstattung in Europa

Felix C. Schweren und Alexander Brink

Korreferat

CSR und der Streit um Freiwilligkeit und Rahmenbedingungen nicht nur bei der Nachhaltigkeitsberichterstattung

Thomas Loew

Rezension

Wirtschaftsphilosophie - Neuer Wein in neuen Schläuchen?

Peter Seele

Rezension

Stark in der soziologischen Konzeption, schwach in der historischen

Umsetzung - Gabriel Abend über die Kontingenz der Wirtschaftsethik Alex Gertschen

Rezension

Es war einmal in Amerika - Ein erstes Überblickswerk der CSR-

Thomas Hajduk 